\title{
Table of legislation
}

\section{INTERNATIONAL INSTRUMENTS \\ WTO}

Agreement Establishing the World Trade Organization 1994 44, 205

Agreement on Agriculture 2, 155, 170, 173, 176

Art 5 133, 176-7

Art $20 \quad \mathbf{1 7 6}$

Agreement on Customs Valuation

140, 185, 209

Agreement on Import Licensing

Procedures 185

Art 2 113, 176

Arts 2-7 222

Art $2.1 \quad \mathbf{1 7 5}, 178$

Art $2.2 \quad 174$

Art 3 133, 177

Art $3.1 \quad \mathbf{1 7 6}$

Art 4 9, 113, 133, 157, 176, 178

Art 4.1(a) 9

Art $4.2 \mathbf{5 7}, \mathbf{1 3 5}, \mathbf{1 8 3}$

Art 4.2(a) 9, 175

Art 4.2(b) $\quad 175$

Art $5.1 \quad \mathbf{1 7 5}$

Art $6 \mathbf{1 7 6}$

Art 7.1 174

Art $7.3 \quad \mathbf{1 7 4}$

Agreement on Textiles and Clothing

$2,49,155,176$

recitals $\mathbf{1 7 6}$
Art $1.1 \quad \mathbf{1 7 6}$

Art $2.20 \quad$ 176-7

Art 6 133, 176-7

Art $8.10 \quad 177$

Anti-Dumping Agreement 2, 31-2,

$42,43,46-7,61-5,115,123-5$, $144,154,156,158-69,171-2$, 175-6, 179-80, 182, 184, 206, 216, 222

Art $1 \quad \mathbf{1 6 3}$

Art 2 163, 222

Art $2.1 \quad \mathbf{1 6 0}$

Art $2.4 \quad \mathbf{1 6 8}$

Art 2.4.2 93

Art 3 133, 144, 157, 167, 222

Art $3.1 \quad \mathbf{1 6 0}$

Art $3.2 \quad \mathbf{1 6 7}$

Art 3.4 163, 168, 171, 175

Art 4 163, 178

Art 5 157, 222

Art $5.1 \quad \mathbf{1 6 3}$

Art $5.2 \quad \mathbf{1 6 3}$

Art $5.8 \quad \mathbf{1 6 3}$

Art $6 \quad \mathbf{1 6 3}, 222$

Art $6.8 \quad \mathbf{1 6 7}$

Art $9.3 \quad \mathbf{1 6 0}$

Art $11.2 \quad 64$

Art $17 \quad \mathbf{1 6 1}$

Art $17.1 \quad \mathbf{1 6 1}$

Art 17.6 2, 31, 45, 46, 59-62, $65,70,78,102,115,123-5$, $138,147,161-6,168$, 179-80, 182, 184

Art 17.6(i) 60-63, 123-4, 162, 164-7, 179-80 
Art 17.6(ii) 61, 64, 123, 125, 165-6, 168-9, 179

Anti-Dumping Code 1979, Art 15.5(b) 41

Dispute Settlement Understanding

2, 31, 46-9, 52, 57, 62, 65, 92-4, 97, 102-3, 105-7, 109, 114,

116-18, 120, 135, 143, 152, 169, 171, 186, 221, 227

Art $1.1 \quad \mathbf{1 6 1}$

Art 3.2 27-8, 30, 47, 83, 91-3, $115,117,119,123,125,148$, 151, 161

Art $3.4 \quad 117$

Art $3.9 \quad 92$

Art $6.1 \quad 31,95$

Art 7.1 105, 161

Art 11 41, 49-53, 57-8, 62-3, $66,78,110-13,115,124$, 134-5, 143, 152-3, 164, 179, 213-14

Art $11.1 \quad 45$

Art $12.1 \quad \mathbf{1 0 5}$

Art $12.7 \quad \mathbf{1 0 9}$

Art $13 \quad 107$

Art 13.1 97, 107

Art 13.2 97, 104, 107

Art 16.4 45, 84, 95

Art $17 \quad 45$

Art $17.4 \quad 95$

Art 17.6 1, 10, 144

Art 19.2 92, 117, 161

Art $22.6 \quad 95$

App 3

para $5 \mathbf{1 0 5}$ para $7 \mathbf{1 0 5}$

Final Act Embodying the Results of the Uruguay Round of

Multilateral Trade Negotiations, Art $4 \mathbf{1 3 3}$

GATS 185, 209, 224

Art VI(2)(a) 185
GATT 1947 31, 37-9, 47, 76, 133, 205

Art XXII 38

Art XXIII 38

GATT 1994 35, 47, 185, 187, 205-9, 212-13, 221

Art I 147, 205-7

Arts I to XXXVII 205

Art III 82, 147, 205-7, 212

Art III:1 206

Art III:2 79, 82, 206

Art III:2.104 $\mathbf{2 0 8}$

Art III:4 85, 206, 208

Art IX:2 92

Art VI 158, 178, 207

Art XI:1 212

Art XIX 174, 178

Art XX 47, 82, 195, 204, 206-8

Art XX(b) 76, 189, 199, 212

Art $X X(d) \quad 199$

Art $X X(g) \quad 47,150$

Art XXI 199

Art XXII, para $1 \quad 38$

Art XXIII, para $1 \quad 38$

Art II(1) 133

Art IX:2 48, 93

Ann 1A $\mathbf{1 5 5}$

Ann 1AA 205

Safeguards Agreement 2, 9, 32, 137, 154-5, 174-8, 182-3

SCM Agreement $\quad \mathbf{2}, \mathbf{5 8}, \mathbf{6 5}, \mathbf{1 3 7}$, 154-7, 159, 169-77, 180-82, 216

Art $1.1 \quad \mathbf{1 7 0}$

Art 1.1(a)(1)(iv) $\mathbf{1 7 0}$

Art $2 \mathbf{1 7 0}$

Art $2.3 \quad \mathbf{1 7 0}$

Arts 3-4 170

Arts 3-7 172

Art $3.1 \quad \mathbf{1 7 0}$

Art $5 \quad \mathbf{1 7 0 ,} 172$

Arts 5-7 170

Art 6 170, 172 
Art $8 \quad \mathbf{1 7 0}$

Art 10 171, 173

Arts 10-22 172, 222

Art 11 133, 157

Arts 11-23 171

Art $11.2 \quad 171$

Art 11.3 171, 173

Art 11.4 157, 171

Art 12 171, 173

Art $13 \quad \mathbf{1 7 1}$

Art $14 \mathbf{1 7 3}$

Art 15 133, 157, 173

Art $15.4 \quad 157$

Art 15.5175

Art $17 \quad \mathbf{1 7 3}$

Art $19 \mathbf{1 7 3}$

Art $19.1 \quad 171$

Art $22 \quad \mathbf{1 7 1}$

SPS Agreement $\quad 35,49,55-6,67$,

74-5, 77, 82, 86, 104, 107,

134-6, 140-41, 185-98, 201-2,

207-11, 213, 220-21

recitals $\mathbf{1 9 5}$

Art 2.1 77, 188

Art 2.2 137, 187-9, 192, 196,

222

Art 2.3 192, 194

Art $3.1 \quad \mathbf{1 8 9}$

Art 3.3 190, 192, 222

Art 5 189, 192

Art $5.1 \quad 32,49,56,59,74,86$, 136-7, 187-9, 192-3, 198, 202, 209-11

Arts 5.1-5.3 190

Arts 5.1 to $5.3 \mathbf{2 2 2}$

Art 5.2 56, 189, 192

Art $5.3 \mathbf{1 8 9}$

Art $5.4 \quad 192$

Arts 5.4 to $5.6 \quad 222$

Art 5.5 137, 194

Art 5.6 59, 188, 190, 192, 194-5
Art $5.7 \quad \mathbf{1 9 0 ,} 198$

Art $5.8 \quad \mathbf{1 8 9}$

Art 11.2 104, 190

Ann A

$\begin{array}{ll}\text { para } 1 & \mathbf{1 8 8} \\ \text { para } 4 & \mathbf{1 9 3}\end{array}$

Subsidies Code $1979 \quad \mathbf{4 1}$

Art $17 \quad 41$

Art $18.1 \quad 41$

TBT Agreement $\quad \mathbf{3 5}, \mathbf{7 7}, \mathbf{8 2}, \mathbf{1 4 0}$,

185-7, 196, 199-204, 207-9,

211-13, 220-21

Art $2.1 \quad 200$

Arts 2.1-2.8 137

Art 2.2 199-204, 211, 222

Art 2.4 200-203, 211

Art 2.5 200, 203

Art 2.9 200, 203

Art 5.1.1 200

TRIPS Agreement $\mathbf{8 5}, \mathbf{1 2 7}, 224$

Understanding on Dispute

Settlement 1979, Art 16

40-41

\section{Non-WTO}

European Convention on Human

Rights

Art 6(1) $\mathbf{2 0}$

Art $10 \quad 23$

International Convention on the Settlement of Investment Disputes between States and Nationals of Other States 1965

Art $25 \quad 24$

Arts 36-49 24

Maastricht Treaty

Art 173 20-21

Art $189 \mathbf{2 0}$

North American Free Trade

Agreement $1992 \quad \mathbf{2 5}$

Art 1904(3) 25 
Ch 19 25-6

Ann $1911 \quad 25$

Statute of the International Court of Justice 1945

Art $36 \quad 24$

Art 36(2) 24

Vienna Convention on the Law of Treaties 1969 31, 61, 64, 92, 114, 116, 125, 149, 166, 216 Art 31 48, 61, 113, 115-16, 148, 162, 165-6, 169 Art 31(1) 63-4, 116 Art 31(3) 149

Art 32 61, 113, 115-16, 148, 162, 165-6, 169

NATIONAL INSTRUMENTS

\section{Australia}

Administrative Appeals Act 1975

$\begin{array}{ll}\text { (Cth) } & \mathbf{1 4} \\ \text { s 25(1) } & \mathbf{1 4} \\ \text { s 33(1) } & \mathbf{1 4} \\ \text { s 35(1) } & \mathbf{1 4}\end{array}$

Administrative Appeals Tribunal Act 1975 (Cth), s 2A 14

Administrative Decisions (Judicial Review) Act 1977 (Cth) 13-14 ss 5-7 13-14

\section{Canada}

Federal Court Act, s 18.1(4) 25

\section{Mexico}

Federal Fiscal Code, Art $238 \quad 25$

\section{United States}

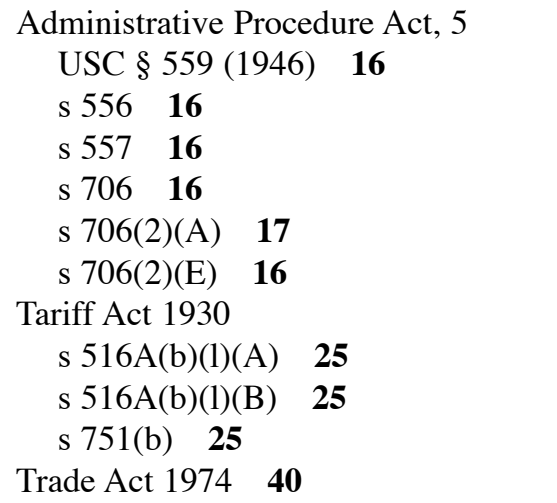


Ross Becroft - 9781781002247 Downloaded from PubFactory at 04/26/2023 02:56: ๑3AM via free access 